\title{
Global challenges posed by the growth of end-stage renal disease
}

\author{
James B. Wetmore ${ }^{1,2}$ and Allan J. Collins ${ }^{1,3^{*}}$
}

\begin{abstract}
Profound challenges confront societies worldwide due to the growth of end-stage renal disease (ESRD). Meeting these challenges requires a sober assessment of how overall global health trends affect ESRD, coupled with an understanding of how progress in the care of ESRD patients is most appropriately measured and monitored. For many affluent countries, incident ESRD rates have stabilized since about the middle of the last decade. For example, the number of new cases in the USA has remained stable at approximately 110,000 per year during this period, while in Japan, growth in annual incident counts appears to have stabilized as of 2012. However, incidence rates rose for many developing countries. An immense "renal replacement therapy (RRT) gap," the difference between the number of people receiving RRT and the number needing it, was recently described, highlighting the magnitude of the difficulties these countries face as they continue to develop. To guide efforts to improve care, broad goals should be established by the worldwide renal health care community. For countries without universal dialysis access, expansion of RRT accessibility should be the first target in order to reduce the RRT gap; for countries that have moved beyond this stage, the goals should include reductions in ESRD incidence rates; all-cause, cardiovascular, and infection-related death rates; and cardiovascular- and infection-related hospitalizations. To inform judgements about progress in this area, measures beyond the traditional standardized mortality ratio are needed. Carefully determining trends over time within a given country, such as is done by the World Health Organization, might prove to be a more useful way to determine progress in combating ESRD and its complications.
\end{abstract}

Keywords: ESRD, Dialysis, Kidney disease, Global health

\section{Background}

Profound challenges confront societies worldwide as a result of the growth of kidney disease in general and of end-stage renal disease (ESRD) in particular. Predictions about likely future increases in the maintenance dialysis population suggest that the burden of providing maintenance dialysis in coming decades will be daunting [1-4]. However, the difficulties faced by each country are likely to be unique, given the national differences in population structure, socioeconomic profile, and culture. Meeting these challenges requires a sober assessment of how overall global health trends affect ESRD, coupled with an understanding of how progress in the care of ESRD patients is most appropriately measured and monitored. Such

\footnotetext{
* Correspondence: acollins@cdrg.org

${ }^{1}$ Chronic Disease Research Group, Minneapolis Medical Research Foundation, 914 South 8th Street, Suite S4.100, Minneapolis, MN 55404, USA

${ }^{3}$ Department of Medicine, University of Minnesota, Minneapolis, MN, USA

Full list of author information is available at the end of the article
}

efforts will likely better prepare ministries of health around the world to make difficult choices as they seek to satisfy competing health priorities.

\section{Overall global health trends}

Trends in ESRD care are intimately linked with broader developments in public health. Fortunately, recent decades have witnessed some welcome developments in the general population. Using the framework of the World Health Organization (WHO), which compiles worldwide data on health outcomes, causes of death can be broadly divided into those resulting from communicable diseases and from non-communicable diseases (NCDs) [5]. Because of the stark differences in socioeconomic status between nations, WHO classifies countries into one of four broad income categories. In developed countries such as the USA and Japan, national bodies also track health outcomes and establish population- 
wide goals, such as those outlined in the US Healthy People 2020 initiative [6].

In developed countries, communicable diseases (in the WHO classification, these include maternal, perinatal, and nutritional conditions) contribute to only a modest percentage of deaths, as might be expected: $<13 \%$ in Japan and $<7 \%$ in the USA, UK, France, Canada, and Australia. As a result, most public health efforts in developed countries are focused on NCDs, and WHO set as its target a $25 \%$ decrease in NCD deaths by 2025 [5]. Fortunately, major strides have been made in addressing NCDs in developed countries, especially in the realm of cardiovascular disease (CVD). For example, between approximately 2000 and 2012, Japan experienced a $30 \%$ decline in CVD deaths in women and a $24 \%$ decline in men; in the USA, corresponding numbers are 27 and $30 \%$. Overall declines of approximately $35 \%$ occurred in the UK, France, Canada, and Australia during this period. Declines in CVD deaths have been so prodigious that cancer is now the leading age-adjusted cause of death in many developed countries [5].

However, the situation varies considerably in countries that are not classified as high-income. For example, in upper-middle income countries such as Brazil, Thailand, and Malaysia, overall rates of NCDs and of CVD are falling-a very welcome development-despite persistently high rates of communicable diseases (11-18\%) relative to more developed countries. China is a special case among this economic group: despite enviably low rates of communicable diseases (5\%, consistent with rates in Western countries), rates of CVD and cancer are rising, perhaps due to unprecedented urbanization and rapidly increasing affluence. Further, among lower-middle and low-income countries, daunting challenges remain: communicable disease rates remain very high (e.g., $25 \%$ for the Philippines, $29 \%$ for India, $37 \%$ for Cambodia, and $67 \%$ for the Democratic Republic of Congo) at the same time that NCD death rates are unchanged (India and Cambodia) or increasing (the Philippines and the Democratic Republic of the Congo) [5].

\section{Health trends in ESRD}

As with NCD- and particularly CVD-related death, some promising developments have occurred regarding ESRD. For many affluent countries such as the USA and Western European countries, incident ESRD rates appear to be relatively stable since about the middle of the last decade, as shown in Fig. 1. However, during this same period, incidence rates rose for many developing countries, such as Bangladesh, Malaysia, Argentina, and Chile, suggesting that the economic status of a country is associated with the development or recognition of ESRD or both.

In a developed country with the largest number of dialysis patients, the USA, incidence rates have been stable since approximately 2003 [7], when the annual percentage increase fell to zero for the first time since 1995 [8].

The number of new US cases has remained stable at approximately 110,000 per year during this period [7]. As shown in Fig. 2a, the incidence rate in 2012, at 359 per million persons, was $6.6 \%$ lower than that in 2003 (385 per million persons) [8], suggesting that the country

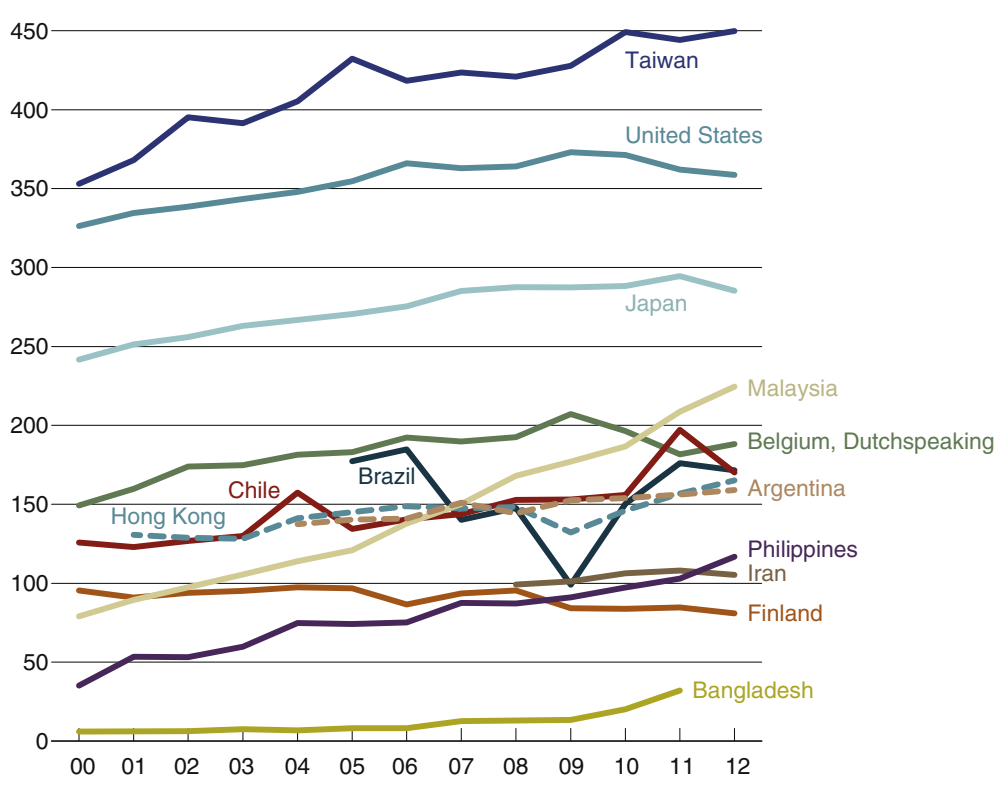

Fig. 1 Incidence rates of end-stage renal disease, by selected country, 2000-2012. Data for 2012, for Iran, and for Taiwan 2009-2012: from the 2014 USRDS AnnualData Report; remaining data: from the 2013 USRDS Annual Data Report 

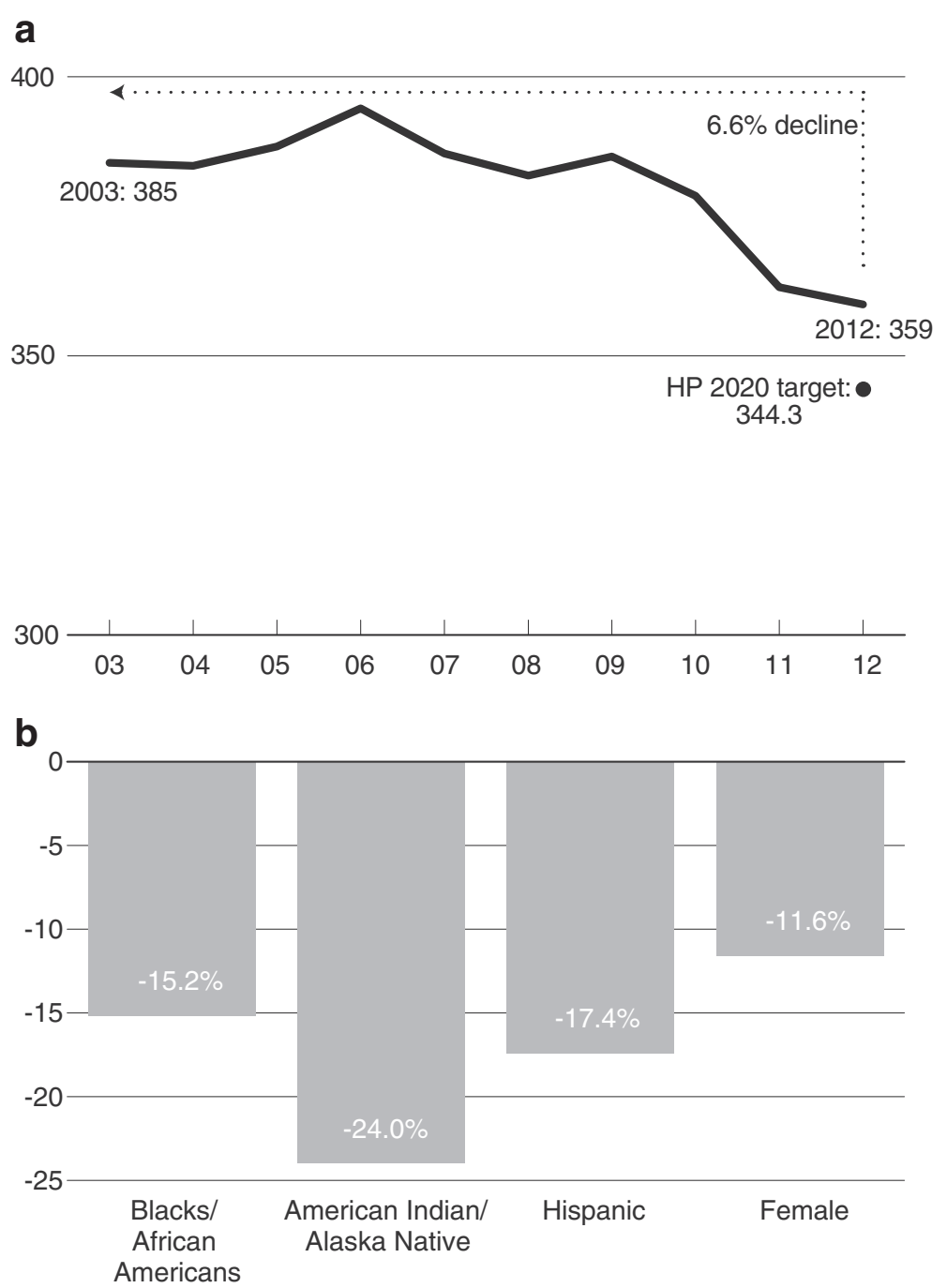

Fig. 2 a Incidence rate of end-stage renal disease, per million population, in the USA; $\mathbf{b}$ percent change in incidence rates of end-stage renal disease, by selected demographic category, in the USA

was making substantial progress towards the Healthy People 2020 goal of 344 per million persons [6].

Perhaps even more impressive, certain traditionally disadvantaged groups experienced higher-than-expected improvements, such as African Americans (15\% decrease), American Indian/Alaskan natives (24\% decrease), Hispanics (17\% decrease), and women (12\% decrease), as shown in Fig. 2b [8]. This is a welcome outcome with implications well beyond the USA, since many other developed countries also have substantial underserved populations at risk for ESRD, such as aboriginal peoples in Canada and Australia and economic and political migrants from Africa, the Middle East, and Asia in Europe [9-13]. However, it is far from clear why incident counts have stabilized: improved control of diabetes, hypertension, and, perhaps, hyperlipidemia may be posited, but whether this is truly the reason is uncertain.

At the same time, life expectancy for patients receiving maintenance dialysis has risen steadily in many developed countries. For example, as shown in Fig. 3, the unadjusted death rate in the USA declined by approximately $20 \%$ between 2003 and 2012 [7]. This corresponds to an increase in the mean survival on dialysis of about 1 year, or about $20 \%$, and an immense number of deaths prevented during that time. Paradoxically, this creates a situation in which improvement in the care of prevalent ESRD patients necessarily means that the absolute number of patients receiving maintenance dialysis at any time will increase, suggesting to the uninformed observer that the "ESRD problem" is worsening. Thus, at present, it is growth in the prevalent, as opposed to the incident, population that 


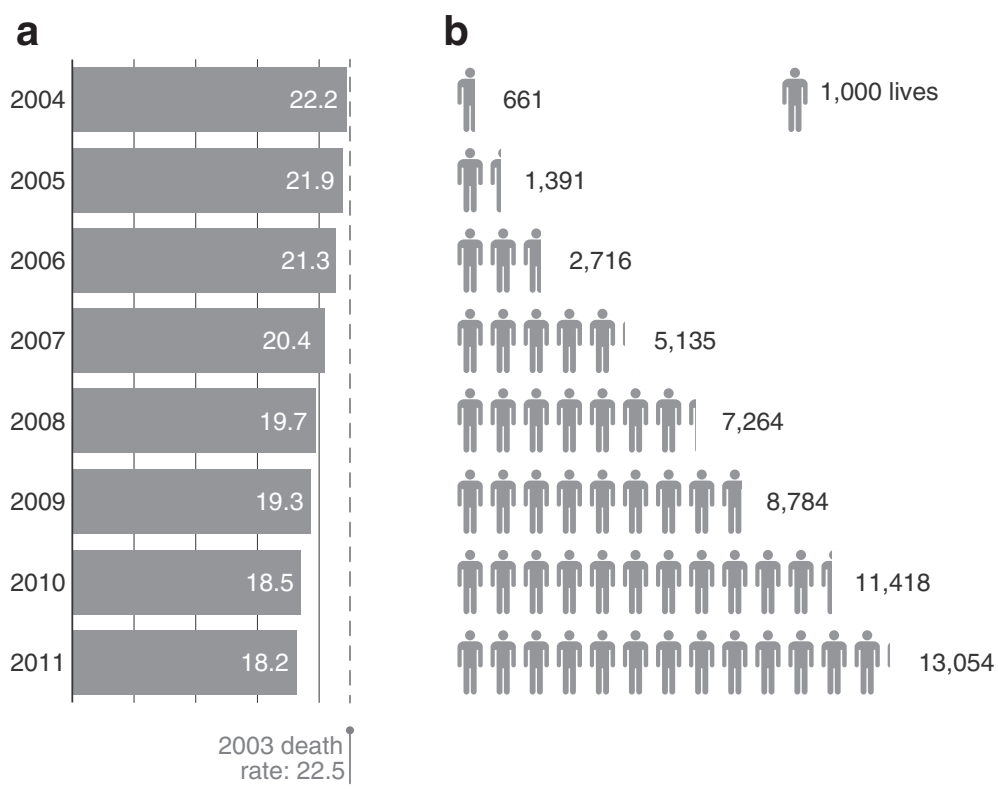

Fig. 3 a Deaths per 100 patient-years, 2004-2011, in US dialysis patients; b number of deaths prevented due to cumulative changes in the death rate in US dialysis patients since 2003

has been the main factor in the growth of the dialysis population in many developed countries. This is particularly true in the USA, where the number of prevalent dialysis patients is expected to grow from approximately 451,000 in 2012 to approximately 632,000 in 2025 [14].

However, the phenomena described above are not universal to all developed countries. In Japan, for example, unique challenges are in evidence. Long the envy of the rest of the world for the survival of its prevalent dialysis patients, Japan has, fortunately, experienced a slowing of growth in the incident counts as of 2012 [15]. Annual percentage change has hovered around zero for the past several years, suggesting that Japan may be approaching a period of stability in annual incident counts that is manifest in other developed countries. However, unlike in some other developed countries, 1-, 5-, and 10-year survival rates have not noticeably improved, and 15- and 20 -year survival rates may have declined [16]. While survival remains much higher in Japan than in other countries, further improvement should always be sought, and public policy planners should be aware that stabilization in the prevalent population is, in reality, a sign that new efforts to improve dialysis survival must be supported. Perhaps technological advancements such as the use of ultrapure dialysis [17-19] might conceivably result in gains in prevalent patient survival. Additionally, the Japanese research community should continue to investigate whether increasing the use of $\beta$-adrenergic blockers, which may benefit some patients $[20,21]$ and which are used in comparatively lower amounts in Japan than in some other countries [22], could provide longevity benefits, especially in patients with heart failure or left ventricular hypertrophy.

Developing countries face a very different set of challenges. Unlike many affluent countries, where access to renal replacement therapy (RRT) is universal or nearuniversal, developing countries must confront the issue of access to RRT, which at present is severely limited. A recent report demonstrates the scope of the problem. Liyanage et al. [1] systematically reviewed data from more than 120 countries and, by hypothesizing that ESRD is related to median age, life expectancy at birth, and gross national income at the national level, estimated age-specific RRT rates in order to calculate the world's "RRT gap" or the difference between the number of people receiving RRT and the number needing it. These investigators demonstrated that, as of 2010, the number of individuals receiving RRT worldwide was projected as 2.6 million, with about $93 \%$ residing in high- or high-middle income countries. This effectively represents a 70-fold prevalence difference between richer and poor countries when population size is considered [2]. The number of individuals on dialysis was projected to more than double by 2030 to 5.4 million, with the largest growth expected in Asia. Perhaps even more informative were the projections of the RRT gap: the authors suggested that only one quarter to one half of individuals who need RRT receive it, meaning that the RRT gap might result in 2.3 to 7.1 million premature deaths.

This finding is conceptually aligned with that of a second study, which estimated an approximately $70 \%$ 
increase in prevalent ESRD patients between 1990 and 2010, fueled by a doubling of the incidence rate [3]. These authors suggested that while changes in population structure might be responsible for one-third of this increase, the main growth is likely due to the increasing provision of RRT by governments and health ministries worldwide. Collectively, these studies suggest that increasing access to dialysis would result in millions more dialysis patients in South America, Africa, India, China, and the rest of Asia.

The worldwide nephrology community and health ministries across the globe must be sober about the substantial economic challenges involved in closing the RRT gap. Providing dialysis to all who need it will require major expenditures in both infrastructure and human capital. Calculating this cost, especially when it is considered in terms of cost-effectiveness, is difficult. Recent small-scale evidence, for example, indicates that provision of RRT in Japan could be cost-effective [23]. However, generating cost-effectiveness estimates is a complex exercise requiring many assumptions that, if incorrect, can lead to overtly misleading findings and indeed can be quite controversial [24-26]. Furthermore, costeffectiveness findings applicable to one economic system are unlikely to apply to another.

A conceptual summary of kidney disease priorities for higher- and lower-income countries is shown in Table 1. Priorities in higher-income countries should focus on improving and refining efforts to detect chronic kidney disease (CKD) in the hopes of delivering therapies to slow its progression, thereby preventing advancement to ESRD. Major efforts should address increasing the number of living related kidney donors to decrease the prevalent dialysis population and, ideally, offer the possibility of preemptive transplant. Efforts to reduce morbidity and mortality, especially cardiovascular and infection-related deaths, should continue apace. Finally, exploration of

Table 1 A conceptual summary of kidney disease priorities for higher- and lower-income countries

\begin{tabular}{ll}
\hline Higher-income countries & Lower-income countries \\
\hline Detect CKD and prevent ESRD & $\begin{array}{l}\text { Continue to target communicable } \\
\text { diseases, which are a risk for AKI }\end{array}$ \\
$\begin{array}{ll}\text { Increase living-related donor } \\
\text { kidney transplants, especially }\end{array}$ & $\begin{array}{l}\text { Establish physical and human } \\
\text { infrastructure to treat CKD and }\end{array}$ \\
for preemptive transplantation & perform transplants \\
Reduced morbidity and & Reduce physical, economic, and cultural \\
mortality in dialysis patients & barriers to transplant \\
Seek novel payment models & $\begin{array}{l}\text { Promote PD and home HD and, with } \\
\text { developed countries, seek ways to make } \\
\text { to reduce costs }\end{array}$ \\
& $\begin{array}{l}\text { dialysis more affordable and less water } \\
\text { use-intensive }\end{array}$ \\
\hline
\end{tabular}

$A K I$ acute kidney injury, CKD chronic kidney disease, ESRD end-stage renal disease, $H D$ hemodialysis, $P D$ peritoneal dialysis methods to control ever-increasing costs, such as the use of capitated fees and bundled payment models [27, 28], must continue. Lower-income countries, in contrast, face some fundamentally different challenges. They must make efforts to reduce communicable diseases that can lead to acute kidney injury (and therefore progressive CKD); attempt to establish the physical health infrastructure, human professional capital, and cultural acceptance necessary to perform both deceased donor and living-related donor kidney transplants; and attempt to develop the ability to deliver lower-cost and less water-intensive dialytic therapies such as peritoneal dialysis and home hemodialysis. As the challenges faced by the lower-income countries are immense, it is likely they will need the assistance of more affluent countries; the developed countries should take the challenges of lower-income countries seriously and seek technological solutions to make dialysis more feasible and affordable, especially in rural areas.

\section{Monitoring progress in kidney disease: elements of an effective framework}

Progress in NCDs and in kidney disease is inextricably linked, albeit in complex ways. The major causes of CKD and, by extension, ESRD, in developed and in the increasingly affluent developing countries are, in large measure, the same causes that contribute to cardiovascular mortality. Hypertension, diabetes, hyperlipidemia, metabolic syndrome, and inflammation, among other mechanisms, contribute to both CVD-related death and to ESRD [29]. Thus, the ESRD population is, in a sense, "contained" within the CVD sphere. As such, kidney disease, including ESRD, has now finally become a target for progress in its own right. In 2011, WHO, via the UN Political Declaration on NCDs, explicitly recognized the threat posed by renal disease, stating, in item 19 , that “... renal, oral and eye diseases pose a major health burden for many countries and that these diseases share common risk factors and can benefit from common responses to non-communicable diseases" [30]. In the USA, the Healthy People 2020 project explicitly lists a series of chronic kidney disease public health targets [31]. Thus, kidney disease is now explicitly receiving attention as the global health challenge that it is.

The question that then arises is how best to guide efforts at improving care. First, certain broad goals should be established by the worldwide renal health care community. For countries without universal dialysis access, expansion of RRT accessibility, especially maintenance dialysis, should be the first target. This would help reduce the immense immediate RRT gap that currently exists. For countries that have moved beyond this stage, the goals should include a reduction of ESRD incidence rates; a reduction in all-cause, cardiovascular, and infection-related death rates; and a reduction in CVD- 
related hospitalizations generally, heart failure-specific hospitalizations specifically, and infection-related hospitalizations. While improvement in death rates and hospitalization rates will, paradoxically, appear to increase the societal burden of ESRD by increasing prevalent counts, the world should continue to capitalize on the improvements that have led to the recent lifespan increases in many developed countries. Concomitantly, efforts to educate the public that this represents at least partial victory, rather than defeat, are needed.

Assuming that countries can embark upon these broad goals, how might progress best be measured? Clearly, each nation should first be compared with itself, as WHO does for communicable diseases and NCDs, since the local social, cultural, and economic milieus vary tremendously by country. Within each country, a useful and commonly used method of comparing outcomes is to generate metrics such as standardized morality ratios (SMRs) and standardized incident ratios, which variously rely on direct or indirect standardization to adjust for case-mix differences [32, 33]. Such an approach can be useful to determine whether and how outcomes vary by geographic region and whether large variations in care exist [34]. This approach is widely used in the USA, but should not be limited to high-income countries, since it would likely prove useful in middle- or even lowerincome countries where economic development is markedly unequal and the effects of increasing affluence and access to care are differentially distributed. This is the case for India and China, where urban and rural health care environments differ substantially within each country. Such comparisons would serve to highlight the immense gaps that exist within a country and the need to address them.

However, additional measures beyond the SMR also have an important role to play. Overall progress cannot be measured by SMRs alone, given that they are by nature cross-sectional and, when used to make comparison rankings, centered at specific values (such as 1.0 or 100) [35]. SMRs are not particularly informative when care is likely to be improving across a population as a whole. An alternative, as used by WHO, is to measure progress over time within a geographic region, in which each region serves as its own (temporal) control. The appeal of this approach is that sites (in this case, countries) that improve over time, but have not yet reached the level of higher-performing locales, would not be "penalized" or disparaged, as can happen when SMRs are the sole metric used to judge care. A framework whereby each site acts as its own control would confer several benefits. First, sites that do not appear to be making sufficient progress over time would become the appropriate focus of efforts to improve progress; targeting such sites might be a more effective way of improving care for the greatest number of patients. Second, because simple adjustment for unmodifiable risk factors such as age, sex, race, and cause of ESRD (as is commonly done when generating SMRs) provides little insight to guide opportunities for improvement, the need to investigate other means of improving care would be highlighted. Third, geographically related factors that are likely to affect care but are not explicitly accounted for in SMR adjustment, including socioeconomic factors, environmental factors such as pollution, and local factors such as barriers to care, idiosyncrasies of care delivery systems, and the local "cultures" of care held by providers, would be inherently accounted for (since these factors are unlikely to change rapidly within a geographic unit) by comparing geographical units to each other over time. Collectively, these insights suggest that a combination of SMRs and time trends may be the most effective way of monitoring and demonstrating progress over time.

How might such a system be used in practice? Perhaps historical trends, over a period of, for example, 5 years might be used to define reasonable goals for improvement over the ensuing 5-year period. Providers would then have a "run-in" period of perhaps 1 year in which to implement quality-improvement efforts designed to address targeted outcomes. The period used to assess improvement would then begin, with each area acting as its own control. Areas that fail to demonstrate progress over time would then be subject to scrutiny in an attempt to improve care for the greatest number of patients.

\section{Conclusions}

Many positive developments in overall health outcomes have occurred over the past decade, but the more affluent countries have been the disproportionate beneficiaries; as a result, much work needs to be done to decrease NCD-related deaths in less-affluent countries. Likewise, more work is required to meet ESRD-related challenges, which vary by country. In the case of the USA, Canada, and Western Europe, managing substantial growth in the prevalent dialysis population is the main difficulty; in Japan, sustaining the stabilization in the incident counts and further improving longevity in prevalent patients should be the main areas of focus; in developing countries, increasing access to dialysis to reduce the RRT gap is the greatest challenge. To judge progress on these fronts, metrics such as the SMR are useful for highlighting geographic and other variations in care where economic, geographic, or other disparities exist, while assessment of trends over time in a given country may be the best way to determine whether overall efforts to improve care are effective. 


\section{Competing interests}

Allan Collins is the Co-Director of the Peer Kidney Care Initiative, which receives funding form 14 US dialysis providers.

\section{Authors' contributions}

Both authors read and approved the final manuscript.

\section{Acknowledgements}

The authors thank Chronic Disease Research Group colleagues Delaney Berrini, BS, for the manuscript preparation, Susan Everson, PhD, for the assistance with the data arrangement and graphical presentation, and Nan Booth, MSW, MPH, ELS, for the manuscript editing.

\section{Author details}

${ }^{1}$ Chronic Disease Research Group, Minneapolis Medical Research Foundation, 914 South 8th Street, Suite S4.100, Minneapolis, MN 55404, USA.

${ }^{2}$ Department of Medicine, Division of Nephrology, Hennepin County Medical Center, Minneapolis, MN, USA. ${ }^{3}$ Department of Medicine, University of Minnesota, Minneapolis, MN, USA.

Received: 24 September 2015 Accepted: 12 November 2015 Published online: 23 February 2016

\section{References}

1. Liyanage T, Ninomiya T, Jha V, Neal B, Patrice HM, Okpechi I, et al. Worldwide access to treatment for end-stage kidney disease: a systematic review. Lancet. 2015;385:1975-82.

2. Coresh J, Jafar TH. Disparities in worldwide treatment of kidney failure. Lancet. 2015;385:1926-8.

3. Thomas B, Wulf S, Bikbov B, Perico N, Cortinovis M, Courville $d$ V, et al. Maintenance dialysis throughout the world in years 1990 and 2010. J Am Soc Nephrol 2015, in press. doi:10.1681/ASN.2014101017.

4. Wetmore JB, Collins AJ. Meeting the world's need for maintenance dialysis. J Am Soc Nephrol 2015, in press. doi:10.1681/ASN.2015060660.

5. World Health Organization. Noncommunicable diseases. Country Profiles. 2014. http://www.who.int/global-coordination-mechanism/publications/ ncds-country-profiles-eng.pdf. Accessed 19 October 2015.

6. U.S. Department of Health and Human Services. Healthy people 2020: leading health indicators development and framework. http://www. healthypeople.gov/2020/leading-health-indicators/Leading-HealthIndicators-Development-and-Framework. Accessed 19 October 2015

7. Saran R, Li Y, Robinson B, Ayanian J, Balkrishnan R, Bragg-Gresham J, et al. US renal data system 2014 annual data report: epidemiology of kidney disease in the United States. Am J Kidney Dis. 2015;66 Suppl 1:S1-305.

8. Weinhandl E, Constantini E, Everson S, Gilbertson D, Li S, Solid C, et al. Peer kidney care initiative 2014 report: dialysis care and outcomes in the United States. Am J Kidney Dis. 2015;65 Suppl 1:S1-140.

9. Young TK, Kaufert JM, McKenzie JK, Hawkins A, O'Neil J. Excessive burden of end-state renal disease among Canadian Indians: a national survey. Am J Public Health. 1989;79:756-8.

10. McDonald SP, Russ GR. Current incidence, treatment patterns and outcome of end-stage renal disease among indigenous groups in Australia and New Zealand. Nephrology (Carlton). 2003;8:42-8.

11. Narva AS. The spectrum of kidney disease in American Indians. Kidney Int Suppl. 2003;83:S3-7.

12. Haysom L, Williams R, Hodson E, Roy LP, Lyle D, Craig JC. Early chronic kidney disease in aboriginal and non-aboriginal Australian children: remoteness, socioeconomic disadvantage or race? Kidney Int. 2007;71:787-94.

13. Martin-Cleary C, Ortiz A. CKD hotspots around the world: where, why and what the lessons are. A CKJ review series. Clin Kidney J. 2014;7:519-23.

14. Allan Collins. Data speaks: what the numbers tell us about previous quality initiatives and options for the future. In: Creating a Culture of Quality conference; 2015: Baltimore, MD. http://esrdnetworks.org/qualityconference-march-2015/speaker-ppt-slides/mon-2-00-data-speaks-a.-collins/ view.

15. Nakai S, Hanafusa N, Masakane I, Taniguchi M, Hamano T, Shoji T, et al. An overview of regular dialysis treatment in Japan (as of 31 December 2012). Ther Apher Dial. 2014;18:535-602.

16. Nakai S, Iseki K, Itami N, Ogata S, Kazama JJ, Kimata N, et al. Overview of regular dialysis treatment in Japan (as of 31 December 2009). Ther Apher Dial. 2012;16:11-53.
17. Lederer SR, Schiffl H. Ultrapure dialysis fluid lowers the cardiovascular morbidity in patients on maintenance hemodialysis by reducing continuous microinflammation. Nephron. 2002;91:452-5.

18. Canaud B, Lertdumrongluk P. Ultrapure dialysis fluid: a new standard for contemporary hemodialysis. Nephrourol Mon. 2012;4:519-23.

19. Asci G, Tz H, Ozkahya M, Duman S, Demirci MS, Cirit M, et al. The impact of membrane permeability and dialysate purity on cardiovascular outcomes. J Am Soc Nephrol. 2013;24:1014-23.

20. Cice G, Ferrara L, D'Andrea A, D'lsa S, Di BA, Cittadini A, et al. Carvedilol increases two-year survivalin dialysis patients with dilated cardiomyopathy: a prospective, placebo-controlled trial. J Am Coll Cardiol. 2003;41:1438-44.

21. Agarwal R, Sinha AD, Pappas MK, Abraham TN, Tegegne GG. Hypertension in hemodialysis patients treated with atenolol or lisinopril: a randomized controlled trial. Nephrol Dial Transplant. 2014;29:672-81.

22. Nakao K, Makino H, Morita S, Takahashi Y, Akizawa T, Saito A, et al. Beta-blocker prescription and outcomes in hemodialysis patients from the Japan Dialysis Outcomes and Practice Patterns Study. Nephron Clin Pract. 2009;113:c132-9.

23. Takura T, Nakanishi T, Kawanishi H, Nitta K, Akizawa T, Hiramatsu M, et al. Cost-effectiveness of maintenance hemodialysis in Japan. Ther Apher Dial 2015, in press. doi:10.1111/1744-9987.12314.

24. Kontodimopoulos N, Niakas D. An estimate of lifelong costs and QALYs in renal replacement therapy based on patients' life expectancy. Health Policy. 2008;86:85-96.

25. Verheijde $J$, Rady MY, McGregor JL. Hypothetical modeling about the cost effectiveness of kidney transplantation for end-stage renal disease: untested assumptions, unaccounted variables and unknown consequences. Health Policy. 2008;88:392-6.

26. Niakas D, Kontodimopoulos $\mathrm{N}$. Is renal transplantation the most cost-effective and preferable therapy for patients suffering from end-stage renal disease or not? Health Policy. 2009;89:329-31.

27. Federal Register. Medicare Program; End-stage renal disease prospective payment system; final rule and proposed rule: CMS-42 CFR Parts 410, 413 and 414. Federal Register 75(155). http://www.gpo.gov/fdsys/pkg/FR-201008-12/pdf/2010-18466.pdf. Accessed 19 October 2015.

28. Sedor JR, Watnick S, Patel UD, Cheung A, Harmon W, Himmelfarb J, et al. ASN end-stage renal disease task force: perspective on prospective payments for renal dialysis facilities. J Am Soc Nephrol. 2010;21:1235-7.

29. Lopez-Novoa JM, Martinez-Salgado C, Rodriguez-Pena AB, Lopez-Hernandez FJ. Common pathophysiological mechanisms of chronic kidney disease: therapeutic perspectives. Pharmacol Ther. 2010;128:61-81.

30. United Nations. Resolution adopted by the General Assembly: Annex, 66th Session, Agenda Item 117. http://www.who.int/nmh/events/un_ncd_ summit2011/political_declaration_en.pdf. Accessed 19 October 2015.

31. U.S. Department of Health and Human Services. Healthy people 2020 topics and objectives: chronic kidney disease. http://www.healthypeople.gov/2020/ topics-objectives/topic/chronic-kidney-disease. Accessed 19 October 2015.

32. Wolfe RA. The standardized mortality ratio revisited: improvements, innovations, and limitations. Am J Kidney Dis. 1994;24:290-7.

33. Van den Broeck J, Brestoff J, Kaulfuss C. Epidemiology: principles and practical guidelines. In: Van den Broeck J, Brestoff J, editors. Statistical estimation. Netherlands: Springer; 2013. p. 417-38.

34. Liu J, Li S, Gilbertson DT, Monda KL, Bradbury BD, Collins AJ. Development of a standardized transfusion ratio as a metric for evaluating dialysis facility anemia management practices. Am J Kidney Dis. 2014;64:608-15.

35. van Gestel YR, Lemmens VE, Lingsma HF, de Hingh $1 H$, Rutten HJ, Coebergh JW. The hospital standardized mortality ratio fallacy: a narrative review. Med Care. 2012;50:662-7. 